\title{
Erratum: Topological superconductivity in Rashba semiconductors without a Zeeman field [Phys. Rev. B 92, 014514 (2015)]
}

\author{
Panagiotis Kotetes (1)
}

(Received 30 April 2020; published 22 May 2020)

DOI: 10.1103/PhysRevB.101.209904

This Erratum concerns the amendment of Eqs. (18) and (33) of the paper. Specifically, the local pairing term $\mathcal{H}_{\Delta}=$ $\int d \boldsymbol{r} \Delta(y)\left[\psi_{\uparrow}^{\dagger}(\boldsymbol{r}) \psi_{\downarrow}^{\dagger}(\boldsymbol{r})+\psi_{\downarrow}(\boldsymbol{r}) \psi_{\uparrow}(\boldsymbol{r})\right]$ appearing in Eqs. (18) and (33) should, instead, have the nonlocal form

$$
\mathcal{H}_{\Delta}=\iint d \boldsymbol{r} d \boldsymbol{r}^{\prime} \Delta\left(y, y^{\prime}\right)\left[\psi_{\uparrow}^{\dagger}(\boldsymbol{r}) \psi_{\downarrow}^{\dagger}\left(\boldsymbol{r}^{\prime}\right)+\psi_{\downarrow}\left(\boldsymbol{r}^{\prime}\right) \psi_{\uparrow}(\boldsymbol{r})\right] .
$$

Despite this modification, the analyses and discussions presented in the paper remain essentially unaltered because Eqs. (23) and (34) of the paper are still recovered even when using the here-derived correct nonlocal pairing term. This is true as long as the Fermi wavelength of the superconductor in the normal phase is much smaller than $L_{y}-b$, which is the case of interest. Equations (23) and (34) essentially originate from the local part of $\Delta\left(y, y^{\prime}\right)$, which is obtained as the limit $\Delta\left(y, y^{\prime} \rightarrow y\right)$. This local part was used in Eqs. (18) and (33) of the paper and, for the projected model, provides essentially the same results as the nonlocal pairing term given the length-scale relation mentioned above. However, considering the local part already from the outset for the full model is problematic. The reason is that, for a local pairing term, the spin degeneracy appearing at $k_{x}=0$ cannot be lifted, thus, not allowing for Majorana bound states. Such persistent degeneracies were discussed in Refs. [1,2] and were more recently revisited in Ref. [3]. In fact, I would like to thank the authors of Ref. [3], for noting the inconsistency between Eqs. (18) and (23) of my paper with regard to this aspect and informing me about it.

In the remainder, I determine the nonlocal pairing term $\Delta\left(y, y^{\prime}\right)$ appearing in the corrected equation above and show that it is consistent with Eqs. (23) and (34) of the paper. I consider the heterostructure depicted below in Fig. 1: which is related to the heterostructures shown in Fig. 4 of the paper. To describe the proximity effect for the semiconductor depicted in Fig. 4 of the paper, I employ the tunnel-coupling Hamiltonian,

$$
\mathcal{H}_{\mathrm{T}}=\int_{-\infty}^{+\infty} d k_{x} \int_{-L_{y} / 2}^{+L_{y} / 2} d y T(y)\left[c_{k_{x}, \sigma}^{\dagger}(y, z=0) \psi_{k_{x}, \sigma}(y)+\text { H.c. }\right],
$$

where the operator $c_{\sigma}(\boldsymbol{r}, z)\left[\psi_{\sigma}(\boldsymbol{r})\right]$ annihilates an electron in the superconductor (semiconductor) at position $(\boldsymbol{r}, z)=$ $(x, y, z)[\boldsymbol{r}=(x, y)]$ with spin projection $\sigma=\uparrow, \downarrow$. In the above, we considered that the semiconductor is quasi-one dimensional, i.e., confined on the $y$ axis. Moreover, I assumed that the heterostructure is translationally invariant in the $x$ direction and performed Fourier transforms of the form $f(x) \mapsto f\left(k_{x}\right)$. The strength of the tunnel coupling $T(y)$ generally depends on the coordinate $y$. Here, I consider the stepwise profile $T(y)=0$ for $y \in[-b / 2, b / 2]$ and $T(y)=T$ otherwise.

The next step is to integrate out the degrees of the freedom of the superconductor, governed by the Hamiltonian,

$$
\begin{aligned}
\mathcal{H}_{\text {superconductor }}= & \int d \boldsymbol{r} \int d z \sum_{\sigma=\uparrow, \downarrow} c_{\sigma}^{\dagger}(\boldsymbol{r}, z) \frac{\hat{\boldsymbol{p}}^{2}+\hat{p}_{z}^{2}-p_{F}^{2}}{2 m} c_{\sigma}(\boldsymbol{r}, z) \\
& +\Delta \int d \boldsymbol{r} \int d z\left[c_{\uparrow}^{\dagger}(\boldsymbol{r}, z) c_{\downarrow}^{\dagger}(\boldsymbol{r}, z)+c_{\downarrow}(\boldsymbol{r}, z) c_{\uparrow}(\boldsymbol{r}, z)\right] .
\end{aligned}
$$

Following standard techniques [4,5], I find that the induced pairing on the semiconductor at energy $E$ and wave-vector $k_{x}$ reads

$$
\Delta_{E, k_{x}}\left(y, y^{\prime}\right)=-\Delta T(y) \mathcal{F}\left(y, z=0 ; y^{\prime}, z=0 ; E, k_{x}\right) T\left(y^{\prime}\right),
$$

where $\mathcal{F}\left(\boldsymbol{r}, z ; \boldsymbol{r}^{\prime}, z^{\prime} ; E\right)$ defines the anomalous Green's function of the superconductor and $\mathcal{F}\left(y, z ; y^{\prime}, z^{\prime} ; E, k_{x}\right)$ defines the respective Fourier transform in the case of a translationally invariant superconductor considered here. I consider $k_{x}=0$ since I focus on the bottom of the conduction band of the semiconductor and obtain

$$
\mathcal{F}\left(y, z=0 ; y^{\prime}, z^{\prime}=0 ; E, k_{x}=0\right)=-\int_{-\infty}^{+\infty} \frac{d k_{z}}{2 \pi} \int_{-\infty}^{+\infty} \frac{d k_{y}}{2 \pi} \frac{e^{i k_{y}\left(y-y^{\prime}\right)}}{\left[\frac{\hbar^{2}\left(k_{y}^{2}+k_{z}^{2}-k_{F}^{2}\right)}{2 m}\right]^{2}+\Delta^{2}-E^{2}} .
$$




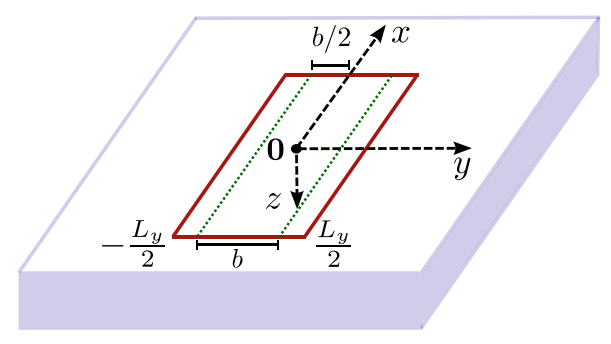

FIG. 1. Schematic of the heterostructure consisting of a bulk superconductor and a semiconductor. The electronic connection and resulting proximity effect of the two materials are blocked along the $y$ axis in the interval $y \in[-b / 2, b / 2]$.

To perform the above integration, I employ the polar coordinates $k_{y}=k \sin \theta$ and $k_{z}=k \cos \theta$ and find

$$
\mathcal{F}\left(y, z=0 ; y^{\prime}, z^{\prime}=0 ; E, k_{x}=0\right)=-\int_{0}^{\infty} \frac{k d k}{2 \pi} \frac{J_{0}\left(k\left|y-y^{\prime}\right|\right)}{\left[\frac{\hbar^{2}\left(k^{2}-k_{F}^{2}\right)}{2 m}\right]^{2}+\Delta^{2}-E^{2}},
$$

where $J_{0}(z)$ denotes the zeroth-order Bessel function of the first kind. At this point, I set $\xi=\hbar^{2}\left(k^{2}-k_{F}^{2}\right) /(2 m)$ and focus on energies much smaller than $\Delta$, i.e., $\Delta^{2}-E^{2} \approx \Delta^{2}$. To proceed, I further consider as it is customary that the Fermi energy of a conventional metallic superconductor is much larger than the superconducting order parameter, which allows for the approximations $k \approx k_{F}$ and $\xi \approx \hbar v_{F}\left(k-k_{F}\right)$, that lead to

$$
\mathcal{F}\left(y, z=0 ; y^{\prime}, z^{\prime}=0 ; E, k_{x}=0\right) \approx-v_{F} J_{0}\left(k_{F}\left|y-y^{\prime}\right|\right) \int_{-E_{F}}^{\infty} \frac{d \xi}{\xi^{2}+\Delta^{2}}
$$

with $v_{F}=m /\left(2 \pi \hbar^{2}\right)$ being the density of states of a two-dimensional electron gas at the Fermi level. I replace the lower limit $-E_{F}$ by $-\infty$ and obtain $\mathcal{F}\left(y, z=0 ; y^{\prime}, z^{\prime}=0 ; E, k_{x}=0\right) \approx-\pi v_{F} J_{0}\left(k_{F}\left|y-y^{\prime}\right|\right) / \Delta$. For small $k_{x}$, I can approximately set $\Delta\left(y, y^{\prime}\right) \approx \Delta_{E, k_{x}=0}\left(y, y^{\prime}\right)$ and conclude to $\Delta\left(y, y^{\prime}\right)=\Delta\left(y-y^{\prime}\right) \approx \Gamma J_{0}\left(k_{F}\left|y-y^{\prime}\right|\right) T(y) T\left(y^{\prime}\right) / T^{2}$ with $\Gamma=\pi v_{F} T^{2}$. The above pairing term can be projected onto the confinement channel wave functions here-denoted $\phi_{n}(y)$. After the length and coordinate system definitions shown in Fig. 1, I have $\phi_{n}(y)=\sqrt{2 / L_{y}} \sin \left[n \pi\left(y / L_{y}+1 / 2\right)\right]$ and find

$$
\Delta_{n m}=\Gamma \int_{-L_{y} / 2}^{+L_{y} / 2} d y \int_{-L_{y} / 2}^{+L_{y} / 2} d y^{\prime} \frac{T(y) T\left(y^{\prime}\right)}{T^{2}} J_{0}\left(k_{F}\left|y-y^{\prime}\right|\right) \phi_{n}(y) \phi_{m}\left(y^{\prime}\right)
$$

Since the Bessel function peaks at $\left|y-y^{\prime}\right|=0$ in which case the proximity-induced pairing term becomes local, I can expand the above in orders of the degree of nonlocality of the pairing which is reflected in the deviation $\ell=y-y^{\prime}$,

$$
\begin{aligned}
\Delta_{n m} & \approx \Gamma \int_{-L_{y} / 2}^{+L_{y} / 2} d y \frac{T(y) \phi_{n}(y)}{T^{2}} \int_{-\Lambda / 2}^{+\Lambda / 2} d \ell J_{0}\left(k_{F}|\ell|\right) T(y+\ell) \phi_{m}(y+\ell) \\
& \approx \Gamma \int_{-L_{y} / 2}^{+L_{y} / 2} \frac{d y}{k_{F}} \frac{T(y) \phi_{n}(y)}{T^{2}}\left\{J_{0}^{(0)} T(y) \phi_{m}(y)+\frac{J_{0}^{(2)}}{k_{F}^{2}}\left[T^{\prime}(y) \phi_{m}^{\prime}(y)+\frac{T(y) \phi_{m}^{\prime \prime}(y)+T^{\prime \prime}(y) \phi_{m}(y)}{2}\right]\right\},
\end{aligned}
$$

where I set $f^{\prime}=d f / d y$ and the integrals $J_{0}^{(n)}=\int_{-k_{F} \Lambda / 2}^{+k_{F} \Lambda / 2} d u u^{n} J_{0}(|u|)$. Note that in the integral over $\ell$, the presence of $J_{0}\left(k_{F}|\ell|\right)$ allowed me to introduce a constant cutoff $\Lambda \ll L_{y}-b$ by virtue of $1 / k_{F} \ll L_{y}-b$. Since, here, $T(-y)=T(y)$ and $\phi_{n}(-y)=$ $(-1)^{n+1} \phi_{n}(y)$, I also find $T^{\prime}(-y)=-T^{\prime}(y), T^{\prime \prime}(-y)=T^{\prime \prime}(y), \phi_{n}^{\prime}(-y)=(-1)^{n} \phi_{n}^{\prime}(y)$, and $\phi_{n}^{\prime \prime}(-y)=(-1)^{n+1} \phi_{n}^{\prime \prime}(y)$. Under the constant-cutoff approximation, these symmetry relations yield $\Delta_{12}=0$. At the same time, the corrections introduced in $\Delta_{11,22}$ by the nonlocal character of the pairing appear to be negligible since they are on the order of $1 /\left(k_{F} L_{y}\right)^{2}$ or smaller. Therefore, Eq. (23) of the paper is, indeed, recovered by only retaining the local part of the nonlocal pairing, and this appears as a good approximation in the limit $1 / k_{F} \ll L_{y}-b$.

These results directly extend to Eq. (34) of the paper, under the assumption which was adopted there, i.e., that the pairing is independent of the $z$ coordinate. The validity of Eq. (34) can be similarly shown by extending the here-discussed Eq. (2) so to include the $z$ coordinate. The associated tunnel-coupling function $T(y, z)$ is once again depending only on the $y$ coordinate, i.e., $T(y, z)=T(y)$. Physically, this holds when the superconducting coherence length is much longer than the nanowire's diameter. 
[1] B. van Heck, S. Mi, and A. R. Akhmerov, Phys. Rev. B 90, 155450 (2014).

[2] F. Pientka, A. Keselman, E. Berg, A. Yacoby, A. Stern, and B. I. Halperin, Phys. Rev. X 7, 021032 (2017).
[3] A. Melo, S. Rubbert, and A. R. Akhmerov, SciPost Phys. 7, 039 (2019).

[4] A. C. Potter and P. A. Lee, Phys. Rev. B 83, 184520 (2011).

[5] J. Danon and K. Flensberg, Phys. Rev. B 91, 165425 (2015). 